\title{
Are gluten-related disorders a new protein aggregation disease?
}

\author{
Veronica Dodero
}

Universität Bielefeld, Germany

https://doi.org/10.17952/35EPS.2018.013

\begin{abstract}
A new hypothesis about the relation of gliadin and its immunodominant33-mer fragment aggregates as triggers of gluten-related disorders was presented.

Gluten-related disorders (1) are a complex group of diseases which involve the activation of the immune system triggered by the ingestion of gluten. These disorders have a high prevalence in western societies around 1-7\% worldwide. The high prevalence is because gluten is present in wheat, rye, barley and some varieties of oats. It is accepted that the incomplete proteolysis of the gluten proteins is responsible for disease. It is assumed that there must be a particular susceptibility in an individual that could trigger this kind of diseases, but parameters have not yet been well clarified. Except for celiac disease (CD), where HLA-DQ genes are of invaluable importance in the diagnosis. Virtually all CD patients (>97\%) carry the coding variants for HLA-DQ2 and /or HLA-DQ8 molecules. Although the genetic predispose is an essential factor in the pathogenesis of $\mathrm{CD}$, it has low predictive value. This is illustrated by the fact that while $30-40 \%$ of the general population carries this genotype, the prevalence of CD worldwide is $1 \%$.
\end{abstract}

Up to now, only a strict gluten-free diet (GFD) improves the clinical symptoms, diminishes the titers of the CD-specific antibodies and finally heals the intestinal mucosa and restores health. CD is an autoimmune enteropathy that may be present not only as intestinal manifestations but also as systemic ones, such as anemia, loss of weight, short stature, osteoporosis, and peripheral neuropathy. Other specific conditions associated with $\mathrm{CD}$ are, for example, Addison's disease, non-Hodgkin lymphoma, neurological disorders and type I diabetes mellitus. Interestingly, it is unknown which are the molecular events that start and persist tissue morphological changes, but the key role of gluten peptides is accepted. In the last years, a high percentage of the population worldwide is opting for a gluten-free diet because they feel "healthy." There is an estimation of self-assessment by adults affecting up to $13 \%$ of the population. In this regard, the Journal Science has recently published a feature article entitled "The war on gluten" (2). It has been shown that gastroenterologists worldwide are confused about the phenomenon of gluten avoiders. Up to know, it is unclear if this is a new diet trend, a style of life or a real public health problem.

Gliadins (3) are immunogenic proteins present in wheat gluten which are classified as $\alpha(25-35 \mathrm{kDa}), \beta(30-35$ $\mathrm{kDa}), \gamma(35-40 \mathrm{kDa})$ and $\omega(55-75 \mathrm{kDa})$ depending on their electrophoretic mobility. These proteins share a similar primary structure consisting of an $\mathrm{N}$-terminal domain, a hydrophobic central domain which is rich in proline, glutamine, and phenylalanine, and, a non-repeating region including cysteine. It is important to mention that the amount and composition of gliadins depend strongly on the wheat variety and the specific cultivar. Also, the structure of the gliadin proteins is itself already complicated due to the heterogeneity of the individual proteins and the wide range of their molecular weights. Humans do not fully degrade these proteins, and after the normal gastric and pancreatic digestion, many peptides remains. The most resistant immunodominant peptide is a 33-mer amino acid fragment, which triggers an autoimmune response related to $\mathrm{CD}(4)$.

From a structural point of view, up to now, the research efforts to the understanding of the pathogenic related molecules were directed towards the identification, quantification, and separation of the components of gluten and connecting them with their pathological role in vitro and in vivo assays. Interestingly, the primary structure of the molecules involved in disease is known, but there is a lack of information about intrinsic behavior, like their folding and molecular organization under physiologically relevant conditions.

Gliadin is self-organized under aqueous physiological conditions: It was demonstrated that when commercial gliadin is dissolved and homogenized in water at $\mathrm{pH} 3.0$ underlow ionic strength, mainly $\alpha$-gliadin remains in the solution self-organized. The self-assembly process is spontaneous and nano-spherical structures, micelletype, are stabilized in the transparent water solution (5). Recently, it was confirmed that even at a dilute concentration 
$(0.1 \mathrm{mg} / \mathrm{ml})$, gliadin is already a colloidal polydisperse system with an average hydrodynamic radius of $30 \pm$ $10 \mathrm{~nm}$. By cryo-transmission electron microscopy (cryo-TEM), it was detected mainly large clusters, but also prolate oligomers were visualized for the first time. Small angle X-ray scattering (SAXS) experiments supported the existence of gliadin prolate/ rod-like structures, suggesting that gliadin dimers are the minimal oligomeric unit organized longitudinally. To understand the basis of the oligomerization, an $\alpha 2$ gliadin model was built, obtained by ab initio prediction and analyzed by molecular dynamics (MD). Combining this model with agg regation predictors a $3 \mathrm{D} \alpha 2$-gliadin model was created showing that there is a major expose surface prone to aggregation at the C-terminus that can be involved in the dimerization process of $\alpha 2$-gliadin (6). The presence of soluble superstructures of $\alpha$-gliadin could explain the high proteolytical resistance, not only due to the high proline content in gliadin primary sequence but also because of inaccessibility of the enzymes due to the aggregation process.

The 33-mer gliadin peptide oligomerizes activating an innate immune response under accumulation conditions. The immunodominant33-mer gliadin peptide remains unprocessed, and it is found in human urine (7). Importantly, this 33 amino acid fragment is directed involved in celiac disease and probably in other immune pathologies associated with gliadin. Although, the immunological response observed in the chronic phase of the celiac disease is well characterized; the cause and the initiation of the inflammatory events are still obscure. From the molecular point of view, the 33-mer behaves as an amphiphile forming nano-aggregates, protofilaments, and lineal plated structures (8). The morphology of the nano-aggregates depends strongly on concentration,and the secondary structure ranges from random, and PPII to parallel beta structure (9). Recently, we reported that only large structures of 33-mer $(>200 \mathrm{~nm})$ induce an innate immune response in macrophages which is mediated by Toll-like receptor (TLR) 4 activation in humans (10). Our investigations showed that if the 33-mer peptide aggregates under accumulation conditions, can trigger a specific innate immune response.

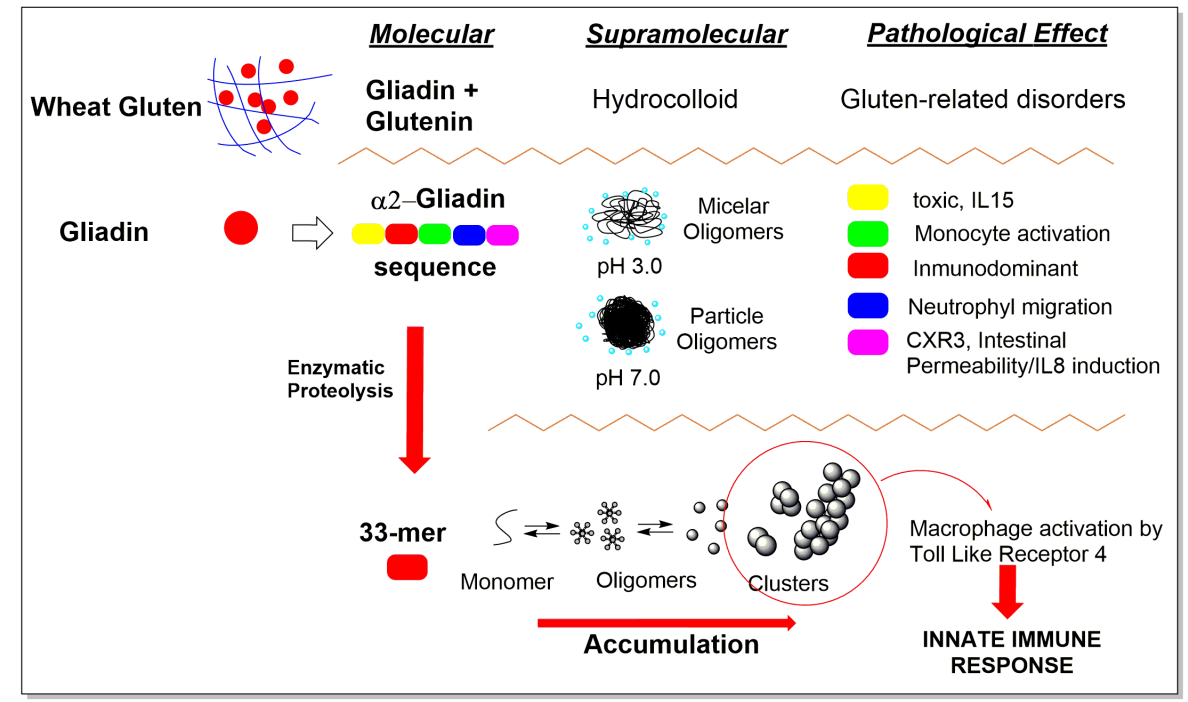

Scheme 1: This cartoon summarizes the relation between molecular structure, supramolecular organization and the pathological effect of gliadin sequence and 33-mer gliadin superstructures.

These findings are the proof of concept that the accumulation and aggregation of this peptide alone can be a hitherto unknown trigger of inflammation. Protein oligomerization and structural change with activation of the innate immune system are hallmarks of other human protein aggregation diseases.

\section{References}

(1) L. M. Lammers, M G. Herrera, V. I. Dodero, Translational Chemistry meets gluten-related disorders, ChemistryOpen 7 (2018) 217.

(2) K. Servick, The war on gluten, Science 360 (2018) 848.

(3) A. V. Balakireva, A. A. Zamyatnin, Jr., Nutrients 8 (2016) 644. 
(4) L. Shan, O. Molberg, I. Parrot, et al., Structural Basis for Gluten Intolerance in Celiac Sprue, Science 297 (2002) 2275.

(5) M. G. Herrera, T. V. Veuthey, V. I. Dodero, Self-organization of gliadin in aqueous media under physiological digestive pHs, Colloids Surf B Biointerfaces 141 (2016) 565.

(6) M. G. Herrera, D. S Vazquez, R. Sreij, M, Dreschler, Y. Hertle, T. Hellweg, V. I. Dodero, Insights into Gliadin oligomerization behavior at digestive pH 3.0, Colloids Surf B Biointerfaces 165 (2018) 363.

(7) M. L. Moreno, A. Cebolla, A. Munoz-Suano et al., Detection of gluten immunogenic peptides in the urine of patients with coeliac disease reveals transgressions in the gluten-free diet and incomplete mucosal healing, Gut (2015) 1.

(8) M. G. Herrera, F. Zamarreno, M. Costabel, H. Ritacco, A. Hütten, N. Sewald, V. I. Dodero, Circular Dichroism and Electron Microscopy Studies in vitro of 33-mer Gliadin Peptide revealed Secondary Structure Transition and Supramolecular Organization, Biopolymers 101 (2014) 96.

(9) M. G. Herrera, L. A. Benedini, C. Lonez, P. L. Schilardi, T. Hellweg, J.-M. Ruysschaert, V. I. Dodero, Self-assembly of 33-mer gliadin peptide oligomers, Soft Matter 11 (2015) 8648.

(10) M. G. Herrera, M. Pizzuto. C. Lonez, K. Rott, A. Hütten, N. Sewald, J-M Ruysschaert, V. I. Dodero, Large Supramolecular Structures of 33-mer Gliadin Peptide Activate Toll-like Receptors in Macrophages, Nanomedicine: NBM 14 (2018) 1417. 